\title{
Entanglement compression in scale space: From the multiscale entanglement renormalization ansatz to matrix product operators
}

\author{
Karel Van Acoleyen, Andrew Hallam, Matthias Bal, Markus Hauru $\odot$, Jutho Haegeman $\odot$, and Frank Verstraete \\ Department of Physics and Astronomy, Ghent University, Krijgslaan 281, S9, B-9000 Ghent, Belgium
}

(Received 14 January 2020; revised 17 July 2020; accepted 14 September 2020; published 15 October 2020)

\begin{abstract}
The multiscale entanglement renormalization ansatz (MERA) provides a constructive algorithm for realizing wave functions that are inherently scale invariant. Unlike conformally invariant partition functions, however, the finite bond dimension $\chi$ of the MERA provides a cutoff in the fields that can be realized. In this paper, we demonstrate that this cutoff is equivalent to the one obtained when approximating a thermal state of a critical Hamiltonian with a matrix product operator (MPO) of finite bond dimension $\chi$. This is achieved by constructing an explicit mapping between the isometries of the MERA and the local tensors of the MPO. In terms of energy scales, our results show that a finite bond dimension MERA is equivalent to introducing both an infrared and an ultraviolet scale, characterizing relevant and irrelevant perturbations on the underlying conformal field theory.
\end{abstract}

DOI: 10.1103/PhysRevB.102.165131

\section{INTRODUCTION}

Arguably, the central problem in the study of extended quantum many-body systems-whether it is in the context of high-energy physics or condensed-matter physics-is the identification of the relevant degrees of freedom. A new perspective on this problem arose with the advent of tensor network states (TNSs) [1]. The crucial feature of the TNS approach is that it directly targets the quantum many-body state and, in particular, its entanglement structure. In this sense TNSs realize a compression or truncation of the exact quantum state in terms of the most relevant entanglement degrees of freedom. In particular, as the virtual (contracted) indices of the tensors encode the entanglement structure, the bond dimension $\chi$ of these indices can be thought of as the systematic control parameter of the entanglement compression achieved by a particular TNS. Given their success thus far, it is desirable to better understand the precise nature of this compression.

To describe critical quantum spin chains, the topic of this paper, there are two distinct tensor networks that offer such compression, namely, matrix product states (MPSs) and the multiscale entanglement renormalization ansatz (MERA). The MPS case underlies the very successful density matrix renormalization group algorithm [2,3], and its success for simulating critical systems stems from the fact that the finite bond dimension $\chi$ approximation plays the role of an infrared (IR) cutoff by effectively adding a small relevant perturbation to the critical system with an amplitude monotonically decreasing as a function of $\chi$. This allows us to construct a theory of finite entanglement scaling for uniform (infinite) MPSs in a vein similar to finite length scaling methods used in exact diagonalization or Monte Carlo [4-8]. Alternatively, matrix product operators (MPOs) have been proven to provide a faithful approximation of thermal states of quantum spin chain Hamiltonians at any finite temperature, with a bond dimension scaling as a power of the inverse temperature, thereby providing an avenue for finite temperature scaling [9-12].

The MERA approach takes a completely different path: the central idea is to define a scale-invariant tensor network for which all correlations decay algebraically by construction, directly aiming at representing the critical ground state [13-18]. The bond dimension $\chi$ of the MERA controls the number of variational parameters, but its physical meaning has never been clarified. The main contribution of this paper is to elucidate the meaning of $\chi$ and to equate it to the bond dimension needed to approximate a thermal state efficiently using an MPO. This solves one of the major open problems in the theory of the MERA and provides a justification for the finite $\chi$ truncation. Notice that while it has been shown that one can construct MERA states directly from the application of the tensor network renormalization (TNR) procedure on the half-finite Euclidean path integral with open physical indices [19], regarding the role of the bond dimension, this merely shifts the question to the role of the bond dimension in TNR.

As demonstrated in [20-22], MERA approximations for critical systems can be understood naturally in scale space: the MERA is a realization of the conformal plane to a strip map, or, equivalently, a map from physical to scale space. We will demonstrate that the half-infinite subsystem density matrix reduces to a thermal (strip) state in scale space, $\rho_{\text {scale }}=e^{-\beta \bar{H}}$, for a Hamiltonian $\bar{H}$ belonging to the same universality class as the original critical Hamiltonian $H$ for which the MERA was optimized. The emerging picture is one where a finite $\chi$ MERA yields a particularly efficient MPO approximation for thermal states. Our numerics indicate that $\chi$ is controlling both an IR and ultraviolet (UV) cutoff of the conformal field theory (CFT) Hamiltonian $\bar{H}$. Furthermore, the (single-site) MERA scaling operator appears as the transfer matrix of this strip state, which relates the MERA procedure for extracting scaling dimensions to Cardy's finite-size scaling [23-25]. Finally, motivated by the MPO compression view, in the last 


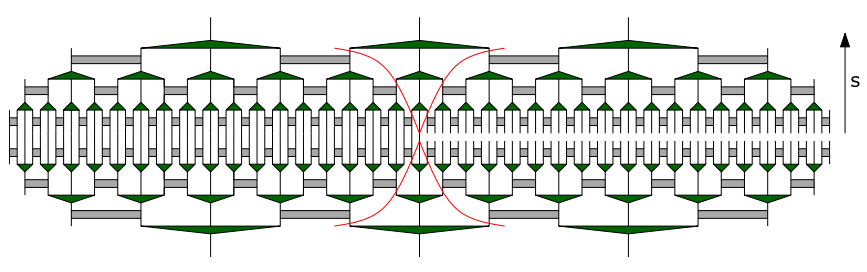

(a)

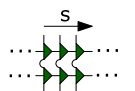

(b)

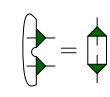

(c)
FIG. 1. (a) The MERA expression for the reduced density matrix on the right infinite half line. (b) The MPO expression for the reduced density matrix in scale space $\rho_{\text {scale }}$, constructed out of the isometries and their conjugates. (c) The corresponding transfer operator for the partition function that is equal to the single-site MERA scaling operator.

part of this paper we propose and implement an algorithm for the construction of isometries and corresponding scaling operators from direct MPO approximations of the thermal state in terms of the original Hamiltonian $H$.

\section{FROM REAL SPACE TO SCALE SPACE, FROM THE PLANE TO THE STRIP}

We consider the half-line density matrix to be realized by a ternary MERA with bond dimension $\chi$, which is numerically optimized on a particular spin Hamiltonian at a critical point. As illustrated in Fig. 1(a), by contracting the ket and bra to the left of the origin, we obtain the reduced density matrix $\rho$ defined on the real-space interval $x \in[0,+\infty]$. All tensors to the left of the causal cone of the origin site drop out due to the unitary and isometric restrictions on the tensors, resulting in the explicit expression $\rho=W \rho_{\text {scale }} W^{+}$. Here $\rho_{\text {scale }}$ is the MPO in Fig. 1(b), with the MPO tensors constructed out of the MERA isometries and their conjugates at the different layers labeled by the renormalization group (RG) $\log$ scale $s$. $W$ is the full isometry from the physical real-space indices on the right half line to the virtual indices in scale space that are cut by the right causal cone. For a critical MERA $\rho_{\text {scale }}$ will be translation invariant up to the first few $s$-dependent isometries that capture the nonuniversal UV behavior of the state.

To understand the bulk properties of $\rho_{\text {scale }}$ we now have to turn to continuum CFT considerations. First, one can verify that $W$ maps local operators at position $x$ in physical space to local operators at the position $s \sim \ln (x) / \ln (3)$ in scale space. The $1 / \ln (3)$ factor arises from a ternary MERA coarse graining three sites to one. Following [20], we are then led to identify $W$ with (a discretized version of) the conformal plane to the strip map,

$$
z \rightarrow w=\frac{\beta}{2 \pi} \ln z
$$

with $\beta=2 \pi / \ln (3), z=x+i t$, and $w=s+i \tau$. From the Hamiltonian perspective $W$ realizes a canonical (isometric) map from the original CFT Hilbert space on the half-line subsystem $x \in[0,+\infty]$ to a new CFT Hilbert space on the full line $s \in[-\infty,+\infty]$, which transforms the original den- sity matrix $\rho=\exp ^{-2 \pi \int_{0}^{+\infty} d x x T_{00}(x)}$ into a translation-invariant Gibbs (strip) state:

$$
\rho_{\text {strip }}=W^{+} \rho W=\exp ^{-\beta \int_{-\infty}^{+\infty} d s \bar{T}_{00}(s)},
$$

where $\bar{T}_{00}(s)$ is the energy density operator on the new Hilbert space.

The above CFT considerations then lead us to the prediction that the bulk density matrix in scale space $\rho_{\text {scale }}$ should converge for increasing bond dimension to a thermal (strip) density matrix $\rho_{\text {strip }}$ with inverse temperature $\beta=(2 \pi) / \ln (3)$ of a CFT Hamiltonian $\bar{H}$ belonging to the same universality class as the original Hamiltonian for which the MERA was optimized:

$$
\rho_{\text {scale }}=\exp ^{-\frac{2 \pi}{\ln (3)} \bar{H}} .
$$

This is the central equation of this paper as it allows us to understand the success of finite $\chi$ MERA approximations in terms of finite bond dimension MPO thermal state compressions. From the work of $[9,10]$ we know that that thermal states of local Hamiltonians can, indeed, be efficiently approximated by an MPO. Approximating a thermal spin chain of length $L$ at temperature $\beta$ with a precision $\left\|\rho_{e x}-\rho_{D}\right\|=\epsilon$ can be achieved with a bond dimension $\chi$ that scales as $O\left((L / \epsilon)^{\beta}\right)$ in the worst case. Numerical studies for specific critical spin chains with central charge $c$ show that this scaling is even more favorable $[11,12]$ and lead to a scaling polynomial in $\beta$, as can also be understood from the result that ground states of critical spin systems can be well approximated using MPSs [26]. Along the lines of [27-29], those results can be extended to the uniform (infinite) limit, and a polynomial scaling is obtained for approximating the local reduced density matrices. Going back to the case of the MERA, we have hence demonstrated that the finite bond dimension MERA tensors approximate the exact tensors efficiently in scale space in the sense that the bond dimension scales polynomially in the precision required, hence justifying the finite bond dimension $\chi$ in the MERA. Below we verify this numerically for MERA simulations on the Ising model. In particular we study the spectrum of the entanglement Hamiltonian $-\ln \rho_{\text {scale }}$ and show that the finite bond dimension introduces relevant (IR) and irrelevant (UV) perturbations to the CFT Hamiltonian, which both decay away (super)polynomially in $\chi$.

Before discussing the numerics let us point out that Eq. (3) ties in nicely with Cardy's seminal results on finite-size scaling for CFTs. From the conformal plane to strip map he shows that the correlation functions on the strip should decay exponentially; in particular the transfer matrix $T$ on the strip (with periodic boundary conditions) should have the spectrum

$$
T=\exp ^{-\frac{2 \pi}{\beta} \Delta},
$$

with $\Delta$ being the scaling dimensions of the local primary operators and their descendants [23-25]. As shown explicitly in Fig. 1(c), upon proper identification of the indices the transfer matrix of $\rho_{\text {scale }}$ indeed precisely reduces to the single-site MERA scaling (super)operator $S$ that is used to extract the scaling dimensions from the MERA simulation according to $S=\exp ^{-\ln (3) \Delta}$.

We have studied the properties of $\rho_{\text {scale }}$ at different MERA bond dimensions $\chi$ for the transverse-field Ising model at its 


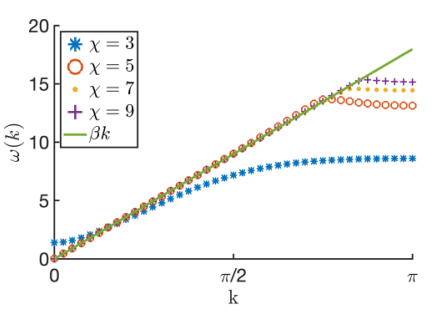

(a)

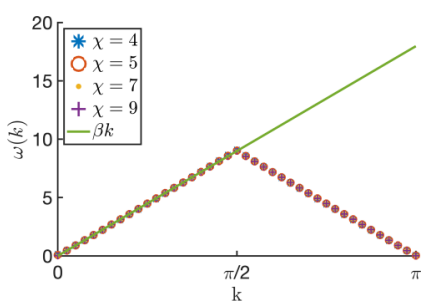

(b)

FIG. 2. (a) The log spectrum of the MPO $\rho_{\text {scale }}$ [Fig. 1(b)], $\omega=-\ln \rho_{\text {scale }}$, in the thermodynamic limit for the bulk isometries optimized on the critical Ising model for different bond dimensions $\chi$. (b) The comparable log spectrum for bulk isometries initialized differently (see text). In each case the blue solid line shows the linear dispersion with gradient $\beta=\frac{2 \pi}{\ln 3}$.

critical point, $H=\sum_{i}-\sigma_{i+1}^{x} \sigma_{i}^{x}-\sigma_{i}^{z}$. First, we numerically optimized all the MERA tensors by minimizing the energy $\langle\Psi|H| \Psi\rangle$ with a new gradient optimization-based algorithm [30], including two nonscale invariant UV layers, imposing the explicit $\mathbb{Z}_{2}$ symmetry of the Ising model $[31,32]$. Then we considered the MPO expression for $\rho_{\text {scale }}$ constructed out of the (scale-independent) bulk isometries [Fig. 1(b)]. Using standard MPS techniques, we then constructed an MPS approximation of the fixed point of the MPO in the thermodynamic limit and used the MPS particle momentum eigenstate ansatz [33] to approximate the one-particle excitations on top of the fixed point.

In Fig. 2 we show the resulting log spectrum, $\omega=$ $-\ln \rho_{\text {scale }}$ for MERA optimizations on the Ising model for different $\chi$. Notice a subtlety involving a MERA gauge choice: depending on this choice one ends up with an isometry that gives rise to a positive or non-positive scaling operator $S$, and a dispersion relation of the type of Fig. 2(a) or 2(b) [34]. These can be interpreted as corresponding to ferromagnetic or antiferromagnetic $\bar{H}$.

From the prediction (3) we would expect a momentum regime with a linear spectrum, $\omega(k)=\beta k$, with $\beta=$ $2 \pi / \ln (3)$. For the different $\chi$ values we indeed find a linear regime in very good agreement with this prediction. As mentioned above, the bond dimension $\chi$ introduces relevant (IR) and irrelevant (UV) perturbations to the CFT result. The relevant perturbation introduces a small mass gap for $k \rightarrow 0$, which for growing $\chi$ decreases as an approximate power law, as can be seen in Fig. 3(a) (red pluses). We note this is not an artifact of our MPS approximation of the fixed point as these results were well converged in the MPS bond dimension. Finite $\chi$ also leads to a divergence from linear dispersion at large $k$. We associate a UV energy scale $\Lambda$ by making the identification, $\omega(\pi)=\beta \Lambda \sin (\pi / \Lambda)$. This choice corresponds to an Ising dispersion relation on a coarse-grained lattice with $O(\Lambda)$ sites blocked into one effective site. In Fig. 3(b) we see that $\Lambda^{-1}$ again decays away rapidly, although the behavior is not clearly polynomial in that case. In the proper scaling regime both relevant and irrelevant perturbations give power corrections to CFT scaling dimensions obtained from finitesize scaling (4) $[24,35,36]$. In fact our numerical results on the scaling dimensions [Figs. 3(c) and 3(d)] point towards a dominant relevant perturbation with scaling dimension 1, e.g.,

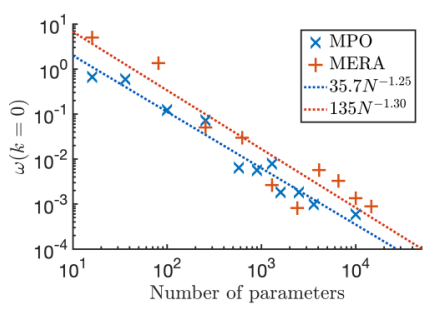

(a)

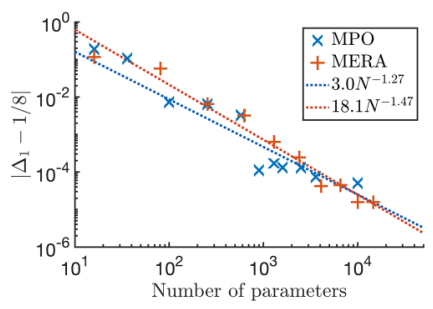

(c)

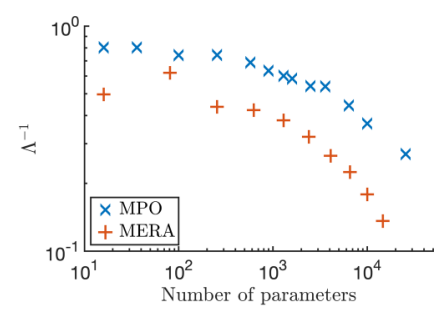

(b)

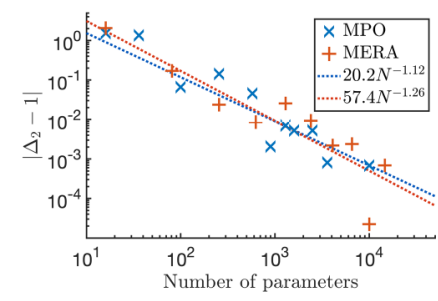

(d)
FIG. 3. For our Ising model simulations with isometries obtained from MERA optimizations (red pluses) and from direct MPO compressions (blue crosses). (a) The IR gap in the dispersion relation. (b) The inverse UV cutoff $\Lambda^{-1}$ in the dispersion relation. (c) and (d) The error in the first two scaling dimensions $\Delta_{i}$. All are given as a function of the number of variational parameters $N$, with $N=\chi^{4}$ in the MERA case $(\chi \in[2,11])$ and $N=16 D^{2}$ in the direct MPO case $(D \in[1,80])$.

a perturbation on the transverse magnetic field in the Ising model [35], as the errors in the scaling dimensions have a polynomial decay in $N$ similar to that of the mass gap $\omega(0)$ [Fig. 3(a)]. This remains true for the higher scaling dimensions, with, e.g., fitted polynomial decays $\sim N^{-1.02}\left(N^{-1.11}\right)$ and $\sim N^{-1.10}\left(N^{-1.03}\right)$ for the next two scaling dimensions $\Delta_{3}$ and $\Delta_{4}$. Here the results in parentheses refer to the direct MPO results that we will discuss now.

\section{FROM THERMAL STATES TO ISOMETRIES AND SCALING OPERATORS}

The results above suggest that a MERA optimization is, in effect, performing an indirect compression of a thermal state for a critical Hamiltonian in terms of an MPO of the particular form in Fig. 1(b). We now consider a specific direct MPO compression scheme. In particular we have followed the approach of [37] by evolving the infinite-temperature state with the Hamiltonian in imaginary time using time-evolving block decimation in a second-order Trotter approximation, with a time step $\Delta t=\frac{\beta}{20000}$ [38]. In this way we have constructed an MPO approximation for $\rho=\exp ^{-\beta H / 2}$, for which the tensors in the proper (left-canonical) gauge are isometric. Upon multiplication of this MPO with its Hermitian conjugate we arrive at an MPO approximation for $\rho=\exp ^{-\beta H}$ of the specific form in Fig. 1(b).

Notice that the resulting isometries are not of the exact MERA form: the two outer lower legs of the isometry have a different bond dimension than the upper leg and center lower leg. The outer legs have a fixed bond dimension $d^{2}=4$ due to the blocking of two lattice sites, each with local Hilbert space dimension $d=2$. The other two legs have a bond dimension $D$ which controls the level of compression. By blocking more 

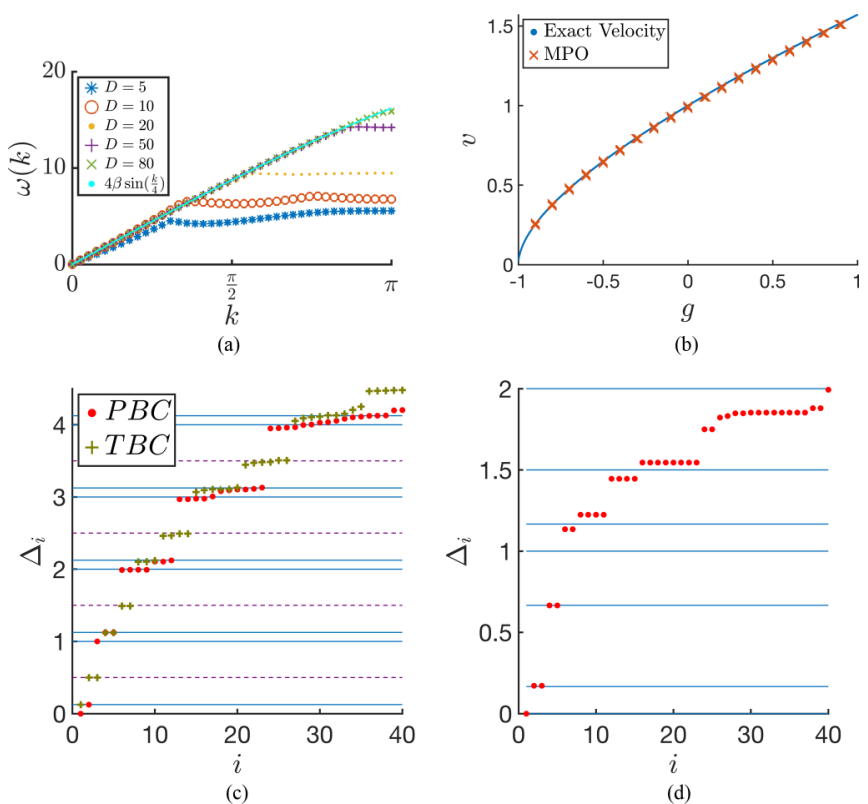

FIG. 4. (a) The log spectra of the MPOs $\omega=-\ln \rho$ for MPO representations of the Ising thermal state at temperature $\beta=2 \pi / \ln 3$ for different $D$. (b) The velocity of the free boson describing the XXZ model for different values of $g$ as calculated from the log spectrum of the $D=30$ MPO; the solid line is the exact result [39]. (c) The Ising scaling dimensions from the MPO transfer operator for periodic (red circles) and twisted (green pluses) boundary conditions (at $D=80$ ). (d) The XXZ (periodic boundary) scaling dimensions for $g=-1 / 2$ (at $D=200)$.

physical sites one could also generate MERA isometries with uniform bond dimension. Another obvious difference is that we now directly target the thermal state of the original microscopic Hamiltonian $H$, in contrast to the MERA case for which the entanglement Hamiltonian $\bar{H}$ shares only the IR properties with the Hamiltonian $H$ on which the MERA is optimized.

In Fig. 4 we show our results from this direct compression scheme for thermal states. Besides the critical Ising Hamiltonian we have also considered the $X X Z$ model. We were, in principle, free to choose $\beta$ but simply stayed with the previous value $\beta=2 \pi / \ln (3)$. Let us first discuss the Ising results. From the generated MPO approximations at different $D$ we obtained the log spectrum Fig. 4(a) in the exact same way as for the MPOs arising from the MERA simulations. The scaling dimensions of Fig. 4(c) were obtained from the transfer (scaling) operator according to (4). We also computed the nonlocal scaling dimensions by adding a twist $\sigma_{z} \otimes \sigma_{z}$ on one of the outer legs of the isometries; see [40] for the same procedure on the MERA scaling operators. In Fig. 3 one can see the IR and UV scale inferred from the direct MPO results [inferred from the results in Fig. 4(a)] together with the errors on the scaling dimensions. Notice that the UV scale $\Lambda$ should now converge to 4 , in correspondence with the exact microscopic Ising result on our blocked lattice. For the MERA case, with growing local dimension $\chi$ we expect $\Lambda \rightarrow \infty$. Barring these specific differences, the overall behavior of the bond dimension dependencies is very similar for both cases, corroborating the view of the MERA optimization performing an indirect MPO compression of a CFT thermal state.

We have also performed simulations on the $X X Z$ Hamiltonian, $H=\sum_{i} \sigma_{i+1}^{x} \sigma_{i}^{x}+\sigma_{i+1}^{y} \sigma_{i}^{y}-g \sigma_{i+1}^{z} \sigma_{i}^{z}$, along the full critical line $|g|<1$. Notice that for this model, in general the velocity $v(g) \neq 1$. This implies a linear dispersion $\omega(k)=$ $\beta v(g) k / 2$ on our (two-site) blocked lattice. From our results on the log spectrum of $\rho$ we can fit a value for $v(g)$ that agrees nicely with the exact result [39], as can be seen in Fig. 4(b). The velocity $v(g)$ also shows up in the scaling dimension relation, which now reads $S=\exp ^{-\frac{4 \pi}{\beta v(g)} \Delta}$. Figure 4(d) shows the $g=-0.5$ results from this scaling operator. The $X X Z$ model is significantly more challenging than the Ising model; this is reflected in the fact that a significantly higher bond dimension is needed to accurately determine its scaling dimensions.

\section{CONCLUSION AND OUTLOOK}

Our results demonstrate that the finite bond dimension $\chi$ approximation of the MERA is of the same nature as the finite bond dimension $\chi$ MPO approximation of a thermal state of a critical Hamiltonian, with $\chi$ controlling both IR and UV perturbations to the CFT. This points to an algorithm for creating critical MERA states from MPO compressions, as the conformal symmetry implies that the very same building blocks of the thermal MPO in scale space also make up the MERA plane to strip map. We have shown how to obtain the isometries; the complete algorithm, of course, also requires a similar construction for obtaining the disentanglers. But notice that the MPO expression in Fig. 1(b) corresponds to a special cut. One can verify that arbitrary cuts lead to MPO expressions for $\rho_{\text {scale }}$ that also involve the disentanglers, giving rise to non-translation-invariant transfer matrices that, depending on $s$, take the form of the single-site scaling operator or the left, center, or right double-site scaling operators. This shows how to obtain the disentanglers: by minimizing the breaking of translation invariance for MPOs corresponding to such general cuts [41]. From the (bulk) disentanglers, in combination with the isometries, one can then also obtain the operator product expansion coefficients [17], overcoming the main limitation of the thermal MPO approach that we presented here.

\section{ACKNOWLEDGMENTS}

We would like to thank L. Vanderstraeten for inspiring discussions. This work is supported by an Odysseus grant from the FWO and has received funding from the European Research Council (ERC) under the European Union Horizon 2020 research and innovation program [Grants Agreement No. 647905 (QUTE) and No. 715861 (ERQUAF) and SIQS].
[1] J. I. Cirac and F. Verstraete, J. Phys. A: Math. Theor. 42, 504004 (2009).

[2] S. R. White, Phys. Rev. Lett. 69, 2863 (1992).
[3] U. Schollwöck, Ann. Phys. (NY) 326, 96 (2011).

[4] T. Nishino, K. Okunishi, and M. Kikuchi, Phys. Lett. A 213, 69 (1996). 
[5] F. Pollmann, S. Mukerjee, A. M. Turner, and J. E. Moore, Phys. Rev. Lett. 102, 255701 (2009).

[6] L. Tagliacozzo, T. R. de Oliveira, S. Iblisdir, and J. I. Latorre, Phys. Rev. B 78, 024410 (2008).

[7] B. Pirvu, G. Vidal, F. Verstraete, and L. Tagliacozzo, Phys. Rev. B 86, 075117 (2012).

[8] B. Vanhecke, J. Haegeman, K. Van Acoleyen, L. Vanderstraeten, and F. Verstraete, Phys. Rev. Lett. 123, 250604 (2019).

[9] M. B. Hastings, Phys. Rev. B 73, 085115 (2006).

[10] A. Molnar, N. Schuch, F. Verstraete, and J. I. Cirac, Phys. Rev. B 91, 045138 (2015).

[11] T. Barthel, arXiv:1708.09349.

[12] B.-B. Chen, L. Chen, Z. Chen, W. Li, and A. Weichselbaum, Phys. Rev. X 8, 031082 (2018).

[13] G. Vidal, Phys. Rev. Lett. 99, 220405 (2007).

[14] G. Vidal, Phys. Rev. Lett. 101, 110501 (2008).

[15] G. Evenbly and G. Vidal, Phys. Rev. B 79, 144108 (2009).

[16] V. Giovannetti, S. Montangero, and R. Fazio, Phys. Rev. Lett. 101, 180503 (2008).

[17] R. N. C. Pfeifer, G. Evenbly, and G. Vidal, Phys. Rev. A 79, 040301(R) (2009).

[18] G. Evenbly and G. Vidal, in Numerical Methods, edited by A. Avella and F. Mancini, Springer Series in Solid-State Sciences (Springer-Verlag, Berlin, Heidelberg, 2013), Vol. 176.

[19] G. Evenbly and G. Vidal, Phys. Rev. Lett. 115, 200401 (2015).

[20] B. Czech, G. Evenbly, L. Lamprou, S. McCandlish, X.-1. Qi, J. Sully, and G. Vidal, Phys. Rev. B 94, 085101 (2016).

[21] G. Evenbly, R. N. C. Pfeifer, V. Picó, S. Iblisdir, L. Tagliacozzo, I. P. McCulloch, and G. Vidal, Phys. Rev. B 82, 161107(R) (2010).

[22] G. Evenbly and G. Vidal, Phys. Rev. B 91, 205119 (2015).

[23] J. L. Cardy, J. Phys. A: Math. Gen. 17, L385 (1984).

[24] J. L. Cardy, Nucl. Phys. B 270, 186 (1986).

[25] J. Cardy, Finite-Size Scaling, 1st ed., Volume 2 (Elsevier, Amsterdam, 1988).

[26] F. Verstraete and J. I. Cirac, Phys. Rev. B 73, 094423 (2006).

[27] N. Schuch and F. Verstraete, arXiv:1711.06559.

[28] Y. Huang, arXiv:1903.10048.
[29] A. M. Dalzell and F. G. Brandão, Quantum 3, 187 (2019).

[30] M. Hauru, M. V. Damme, and J. Haegeman, arXiv:2007.03638.

[31] S. Singh, R. N. C. Pfeifer, and G. Vidal, Phys. Rev. B 83, 115125 (2011).

[32] S. Singh and G. Vidal, Phys. Rev. B 88, 121108(R) (2013).

[33] J. Haegeman, B. Pirvu, D. J. Weir, J. I. Cirac, T. J. Osborne, H. Verschelde, and F. Verstraete, Phys. Rev. B 85, 100408(R) (2012).

[34] In our simulations, depending on the initial random seed in the numerical optimization, we end up with isometries giving rise to two different types of dispersion relations (except for $\chi=3$ ): the ferromagnetic type [Fig. 2(a)] or the antiferromagnetic type [Fig. 2(b)], with only a positive scaling operator $S$ in the former case. By applying a $\mathbb{Z}_{2}$ transformation on the upper leg of the isometry we can switch between both cases. Using the $\mathbb{Z}_{2}$ symmetry of the tensors, we can show that this is a MERA gauge transformation, i.e., a transformation on the MERA tensors that leaves the actual physical state invariant. From the same $\mathbb{Z}_{2}$ symmetry of the tensors one can also show that this transformation shifts the momentum of the MPO eigenstates: $k \rightarrow k+m \pi$, with $m=(0,1)$ being the $\mathbb{Z}_{2}$ quantum number of the state. This then explains the $k>\pi / 2$ part of the second case [Fig. 2(b)] as arising from the $m=1$ negative momentum branch of the first case. This generalizes to more general group symmetries: contracting an element of the group $U_{g}$, with $g$ in the center of the group, on the upper leg of the symmetric isometry, shifts the momentum of the MPO eigenstates accordingly, while leaving the physical MERA state invariant.

[35] M. Henkel, J. Phys. A: Math. Gen. 20, 995 (1987).

[36] P. Reinicke, J. Phys. A: Math. Gen. 20, 4501 (1987).

[37] F. Verstraete, J. J. García-Ripoll, and J. I. Cirac, Phys. Rev. Lett. 93, 207204 (2004).

[38] G. Vidal, Phys. Rev. Lett. 93, 040502 (2004).

[39] T. Giamarchi, Quantum Physics in One Dimension, International Series on Monographs on Physics Vol. 131 (Clarendon Press, Oxford, 2004).

[40] G. Evenbly, P. Corboz, and G. Vidal, Phys. Rev. B 82, 132411 (2010).

[41] K. Van Acoleyen et al. (unpublished). 